\title{
Investigation of metabolites accumulation in medical plant Gentiana rigescens during different growing stage using LC-MS/MS and FT-IR
}

Yu Pan 1,2, Ji Zhang ${ }^{2}$, Yan-Li Zhao ${ }^{2}$, Yuan-Zhong Wang ${ }^{2 *}$ and Heng-Yu Huang ${ }^{1 *}$

\begin{abstract}
Background: Gentiana rigescens, an important medicinal plant in China, has been widely cultivated in Yunnan province, China. Previous studies were focused on analysis and determination of the metabolites isolated from this species, the accumulation of these metabolites during growth period are not yet clear. In this study, samples for the experiments were obtained by tissue culture. FT-IR and LC-MS/MS method were performed to distinguish the variation on the major metabolites in G. rigescens during growing stage when combined with chemometrics.

Results: Methodology validations were all within the required limits. The metabolites were visually different in tissue culture samples and mature plants. The diversity of metabolites increased proportionally with plant growth. The quantitative analysis showed the content of gentiopicroside was significantly vary during different growing stage. The highest content of gentiopicroside $(122.93 \pm 7.01 \mathrm{mg} / \mathrm{g})$ was detected in leaf of regenerated plantlet, whereas its content in root significantly increased along with underground parts growth. Moreover, flavonoids mainly distributed in aerial parts showed potential competitive relationship during plant growth.
\end{abstract}

Conclusion: The distribution and accumulation of metabolites are associated with different parts and plant growth, which provide potential evidences for the rational application and exploitation of G. rigescens.

Keywords: Gentiana rigescens; Tissue culture; Metabolites variation; LC-MS/MS; FT-IR

\section{Background}

Gentiana rigescens Franch belonging to Gentianaceae is an important medicinal plant in China for treatment of hepatitis, jaundice and dysentery. The root and rhizome of this plant has been officially documented in Chinese Pharmacopoeia as one of raw materials of Gentianae Radix et Rhizoma (Longdan in Chinese), a hepatoprotective agent (State Pharmacopoeia Commission 2010). According to ethnobotanical information, its aerial parts are also used as folk medicine for treatment of fever and rheumatic arthritis or as antiophidica, when prepared with vegetable oil. The phytochemistry and bioactivities of G. rigescens have been intensively studied. More than 100 secondary metabolites with different activities like hepatoprotective, anti-inflammatory, antioxidant and

\footnotetext{
*Correspondence: boletus@126.com; hhyhhy96@163.com

${ }^{2}$ Institute of Medicinal Plants, Yunnan Academy of Agricultural Sciences, Kunming, China

${ }^{1}$ College of Traditional Chinese Medicine, Yunnan University of Traditional Chinese Medicine, Kunming, China
}

neuritogenic growth have been isolated from this plant (Gao et al. 2010a, 2010b; Xu et al. 2006, 2007, 2009a; Wang et al. 2012). Among them, iridoid glycosides is the most abundant components especially gentiopicroside which content is more than $4.5 \%$ and serve as major active ingredient and standard for quality control (Jiang et al. 2005; Pan et al. 2015a; Wang et al. 2012).

As one of well-known traditional Chinese medicine with remarkable medicinal functions, the wild resources have been under heavy threat owing to human activities and environmental pollution. Although G. rigescens have been extensively planted in Yunnan, some disadvantages on cultivation such as continuous cropping obstacle, time-consuming and laborintensive, etc. result in decline of production and quality. Fortunately, plant tissue culture is conducive to the accumulation of biomass and metabolites, in particular with individual metabolites, which amount is multifold higher than control group when treated with appropriate elicitors (Chuang et al. 2014; Huang et al. 2014; Kuzovkina et al. 2014; Kumari 
et al. 2015; Marsh et al. 2014; Su et al. 2014). However, the efficacy of medical plant, to a large extend, are attributed to synergistic effect of a number of metabolites. The amount of individual metabolites is significantly increased, which might lead to different pharmacological activities and therapeutic effects.

On the other hand, previous studies (Jiang et al. 2005; Pan et al. 2014, 2015a; Wang et al. 2012) on metabolites of G. rigescens were most focused on quality assessment, phytochemistry, pharmacology, etc. However, metabolites are not only the efficacious properties for maintaining human health, but also play an important role for resistance to abiotic and biotic threats during plant growth (Hall et al. 2008; Wink 2003). To our best knowledge, the accumulation and variation of metabolites in G. rigescens have not yet clear.

Currently, analysis of metabolites based on separation technologies such as gas chromatography-mass spectrometry ( $\mathrm{Hu}$ et al. 2014), liquid chromatography coupled with photodiode array detector ( $\mathrm{Yu}$ et al. 2014), mass spectrometry detector (Won et al. 2014) and nuclear magnetic resonance spectroscopy (Hilbert et al. 2015) can rapidly provide complex chemical information and clarify the similarities and differences of bio-samples when combined with chemometrics. However, comprehensive chemical information on metabolites cannot be analyzed in a single chromatogram. Fourier transform infrared spectroscopy (FT-IR) enables to rapid reflect holistic molecular structure-analyte relationships, which is considered as a well-established and non-destructive method for analysis of bio-sample, whereas it fails to recognise the variation of specific compound in sample due to the limited specificity and sensitivity (Karoui et al. 2010; Lohumi et al. 2014; Zhao et al. 2014).
In this study, the variation on distribution and accumulation of metabolites in G. rigescens are investigated based on plant tissue culture. Individual parts of sample during different growing stage are subjected to targeted and non-targeted analysis using FT-IR and liquid chromatography tandem mass spectrometry (LC-MS/MS). Moreover, the biosynthetic pathway of iridoid glycosides is also discussed. The combinative comparison approach can reflect the overall chemical difference during different growing stage, which may provide the useful information for reasonable utilization of resources.

\section{Methods}

\section{Materials and chemicals}

Tissue culture materials provide by Dr. Heng-Yu Huang (College of Traditional Chinese Medicine, Yunnan University of Traditional Chinese Medicine) were established using leaves of G. rigescens. The operations of tissue culture were completed on a super-clean bench. The plantlets were grown on Murashige-Skoog (MS) medium supplemented different concentration of indoleacetic acid (IAA), zeatin(ZT), dichlorphenoxyacetic acid (2,4-D), kinetin (KT) and 6-benzyladenine (BA) during different stage. The processed of tissue culture is present in Fig. 1. The culturing conditions were incubated at $23 \pm 2{ }^{\circ} \mathrm{C}$ under cool white fluorescent light at 1500-2000 lx under $10 \mathrm{~h}$ per day.

$\mathrm{KBr}$ (specpure) was purchased from Tianjin FengChuan Fine Chemical Research Institute (Tianjin, China). Methanol and formic acid (assigned purity $>98 \%$ ) were LC grade and purchased from Thermo Fisher Scientific (USA) and Dikmapure (USA), respectively. Water was purified to 18.25 $\mathrm{M} \Omega$ using Milli-Q system from Millipore (USA). All other chemicals for extraction were analytical grade. The standard compounds (1. loganic acid, 2. swertiamarin, 3.

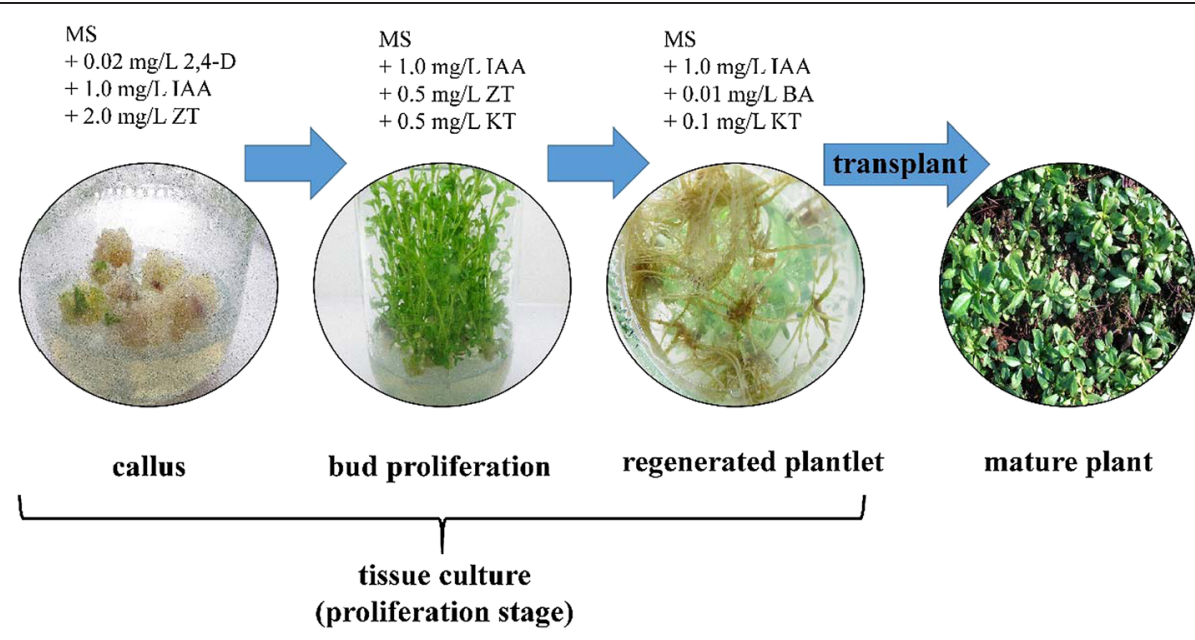

Fig. 1 Tissue culture flowchart of G. rigescens 
gentiopicroside, 4. sweroside, 5 . isoorientin and $\mathbf{6}$. isovitexin) (Fig. 2) were provided by Chinese National Institute for the Control of Pharmaceutical and Biological Products (Beijing, China). Their assigned purity were all $>98 \%$.

\section{Apparatus}

Tablet press (YP-2, Shanghai Shanyue instrument Inc) was used to press powder into thin samples. The FT-IR spectrometer (PerkinElmer, USA) equipped with a DTGS detector. IR spectra were recorded from the accumulation of 16 scans in $4000-400 \mathrm{~cm}^{-1}$ range with a resolution of $4 \mathrm{~cm}^{-1}$.

Separation, quantitation and quantification of metabolites were performed on a Shimadzu Nexera UHPLC tandem mass spectrometry (LCMS-8030, Shimadzu, Japan) equipped with a Shim-pack XR-ODS III $(75 \times 2.0 \mathrm{~mm}$, $1.6 \mu \mathrm{m})$ column, UV detection and triple quadrupole mass spectrometer via an electrospray ionization (ESI) interface. The mobile phase consisted of $0.1 \%$ formic acid in water (A) and methanol (B) was applied at a flow rate of $0.35 \mathrm{~mL} / \mathrm{min}$ with gradient as follow: initial, $13 \% \mathrm{~B}$; 0.31 $7.00 \mathrm{~min} 20 \% \mathrm{~B}$ linear, 7.01-13.00 min $46 \%$ B linear, 13.01-16.50 min $83 \%$ B linear; followed by a final increase to $90 \%$ in $1 \mathrm{~min}$. After a $3 \mathrm{~min}$ wash, the column was reconditioned at $13 \% \mathrm{~B}$ for $3 \mathrm{~min}$ to prepare for the next injection. Injection volume and column temperature were $1 \mu \mathrm{L}$ and $40{ }^{\circ} \mathrm{C}$, respectively. The detection wavelength was set at $242 \mathrm{~nm}$, where all the standards and UPLC profiling showed a satisfactory performance.

High-resolution electrospray ionization mass spectrometry was performed using a Agilent G6550 QTOF (Agilent technologies Santa Clara, CA, USA) equipped with an ESI inter-face. Mass spectra were acquired in both positive and negative modes over the range $m / z$ 100-1000. The capillary voltages were set at $3000 \mathrm{~V}$ (positive mode) and $2700 \mathrm{~V}$ (negative mode), respectively, and nozzle voltage was $300 \mathrm{~V}$. Sheath gas and drying gas were nitrogen at a flow rate of 3.0 and $14.0 \mathrm{~L} / \mathrm{min}$, respectively, nebulizer pressure was 20 psi. The precise molecular mass was determined by the accurate-mass data of the TOF analyzer within a reasonable degree of measurement error, normally with mass errors below $5 \mathrm{ppm}$ in routine analysis, which was sufficient to verify the elemental compositions of the known constituents in G. rigescens.

The quantification of the targeted compounds with low concentration was carried out on multiple reaction monitoring (MRM). The settings of MRM were autooptimized by Labsolutions software (Shimadzu, Japan). The triple quadrupole mass spectrometer parameters were set as follows: nebulizing gas and drying gas were nitrogen at a flow rate of 3.0 and $15.0 \mathrm{~L} / \mathrm{min}$, respectively; the interface voltage was set to $4.5 \mathrm{kV}$; desolvation line (DL) temperature was $250{ }^{\circ} \mathrm{C}$ and the heat block temperature was $400{ }^{\circ} \mathrm{C}$. Reference solutions containing ions of $m / z 503.15$ and 1004.60 were continuously introduced into the MS system during the analysis procedure to ensure the accuracy of the measured mass.

\section{Sample preparation}

Fresh samples were dried at $60{ }^{\circ} \mathrm{C}$ and ground into fine powder. Then, $2 \mathrm{mg}$ sample was blended with $200 \mathrm{mg}$ $\mathrm{KBr}$ powder, ground again and pressed into a tablet. The FT-IR spectra of all samples were collected three times after by subtraction of KBr pellet background.

Sample preparation was based on our previous work (Pan et al. 2015b). An accurately weighed sample powder

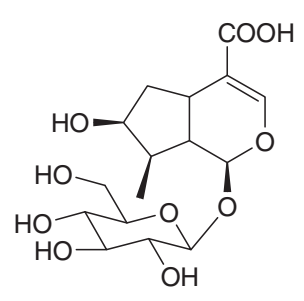

1 loganic acid

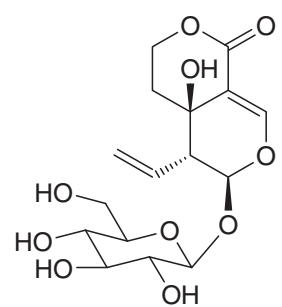

2 swertiamarin

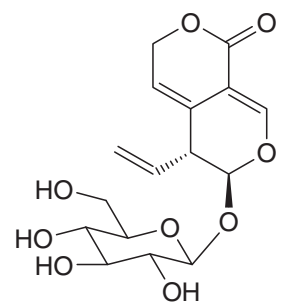

3 gentiopicroside<smiles>C=C[C@H]1C2CCOC(=O)C2=CO[C@H]1OC1OCCC(O)C(O)C1(O)CO</smiles>

4 sweroside<smiles>O=c1cc(-c2ccc(O)c(O)c2)oc2cc(O)c(C(O)OC(O)C(O)CO)c(O)c12</smiles><smiles>O=c1cc(-c2ccc(O)cc2)oc2cc(O)c(C(O)OC(O)C(O)C(O)CO)c(O)c12</smiles>

Fig. 2 Index compounds of G. rigescens. (1. loganic acid; 2. swertiamarin; 3. gentiopicroside; 4. sweroside; 5. isoorientin; 6. isovitexin) 
(0.1 g) was extracted by ultrasonication with $7 \mathrm{~mL} 80 \%$ methanol for $35 \mathrm{~min}$. The extract solution were filtered through a paper filter. Then, the filtrate were stored at $4{ }^{\circ} \mathrm{C}$ and filtered through a $0.22 \mu \mathrm{m}$ membrane filter before injection into the LC system for analysis. Injection volume was $1 \mu \mathrm{L}$.

\section{Data analysis}

The raw FT-IR spectroscopy were processed by Omnic 8.0 (Thermo Fisher Scientific, USA). The peaks of UV and mass data were picked and filtered by Labsolutions software (Shimadzu, Japan). Principal component analysis (PCA), an unsupervised chemometric approach for classification, was exploited to optimize the complex data set for reflecting relationships among different samples. The PCA was performed by software SIMCA$\mathrm{P}^{+} 10.0$ (Umetrics AB, Sweden).

\section{Results}

Comparative analysis of $G$. rigescens in different growing stage

FT-IR and LC-MS/MS were used to investigate the metabolites variation in both integrity and detail on chemical information in different plant part and growing stage. There are few visual differences in averaged FT-IR spectra of different sample. In order to explore the relationships between metabolites and plant growth, the 1800-600 $\mathrm{cm}^{-1}$ region of the FT-IR spectral data without the interferences of $\mathrm{CO}_{2}$ and $\mathrm{H}_{2} \mathrm{O}$ were subjected to PCA. A two-dimension (2D) scores plot $(\mathrm{PC} 1 \times \mathrm{PC} 2)$ was constructed from a data matrix $(623 \times 54)$ by PCA which could visually reflect the similarity between IR spectra and samples in this plot where the closer the points, the more similar the spectral data. In PCA, the first and second principal components cumulatively accounted for $92.8 \%$ of the total variance, which suggested that the former two principal components could explain the proportion of the experimental data. In Fig. 3, sample of mature plant and samples during proliferation stage were explicitly separated into two groups. However, samples in the corresponding groups were crossed and could not be classified according to their plant parts or growing stage. These results implied that the whole metabolome based on FT-IR spectra were significant different, whereas these the detailed variation failed to be monitored especially for content of individual metabolites.

Moreover, a targeted method based on LC-MS/MS was designed for monitoring variations of iridoid glycosides and flavonoids. The $80 \% \mathrm{MeOH}$ extracts of $\mathrm{G}$. rigescens were injected into LC-MS/MS system for analysis. As shown in Fig. 4a-i, peak numbers and their peak areas were visually different in samples, especially for peaks a, 3, 5, 6, b and c, which indicated the accumulation of metabolites varied with plant growth. For example, peak b and c were only found in leaf of mature plant. Furthermore, peak a is one of characteristic marker in chromatogram of callus, whereas it could not detected in chromatogram of mature plant.

Except for peak 1-6 which can be unambiguously identified by standard compounds, other peaks in chromatogram are still unknown. In order to further clarify metabolites variations during growing stage, peak a-g (Additional file 1: Table S1), characteristic markers in chromatogram of samples, were tentatively identified via matching the mass data (high revolution data and MS/MS spectra) with published works on chemical compounds isolated from G. rigescens. According to comparison of mass data of standard compounds, peak

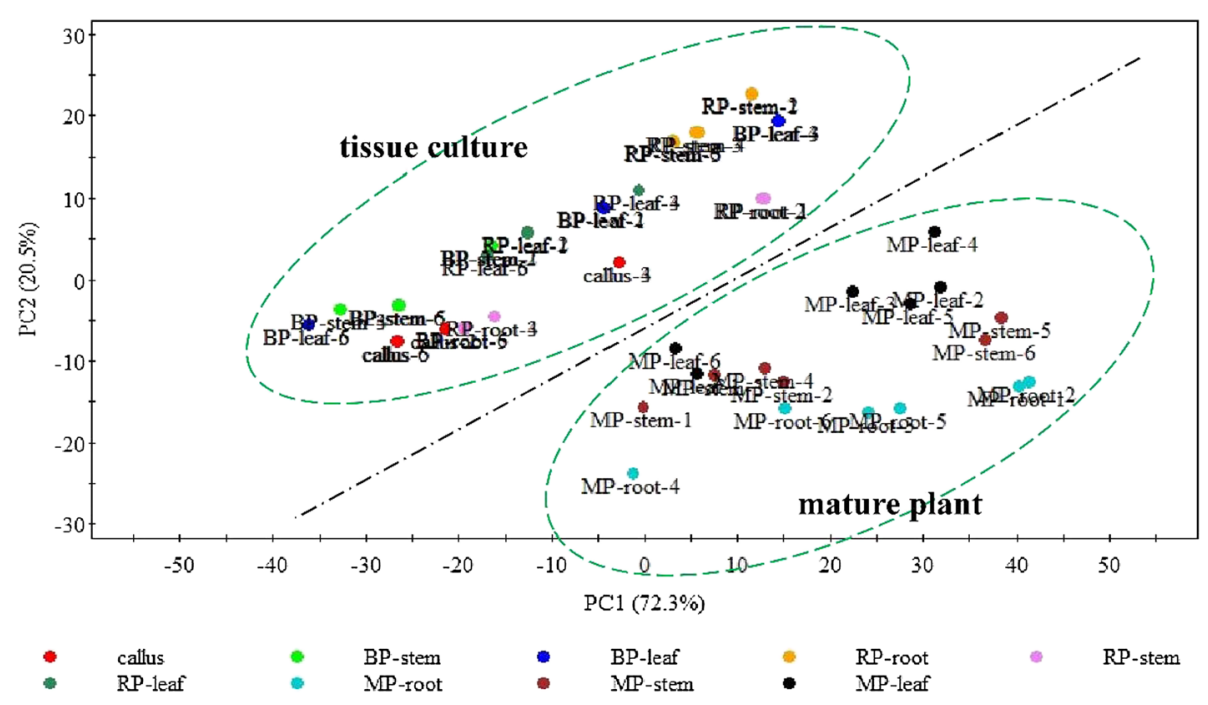

Fig. 3 The PCA scores plot of samples during different stage based on FT-IR 
(a) $\mathrm{mv}$

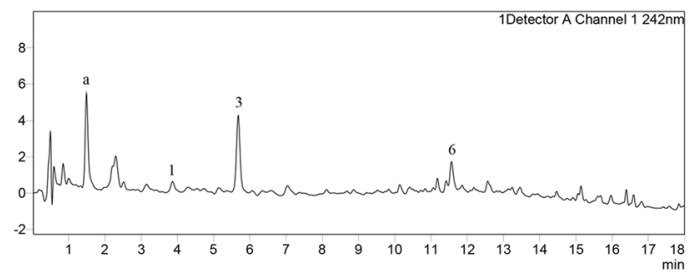

(c) $\mathrm{mv}$

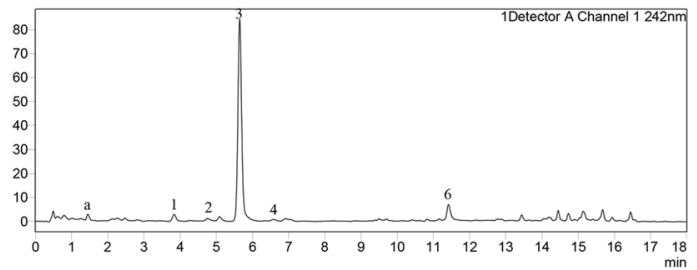

(e) $\mathrm{mv}$

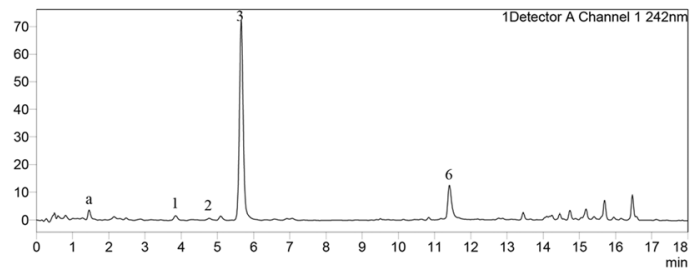

$(\mathrm{g}) \mathrm{mv}$

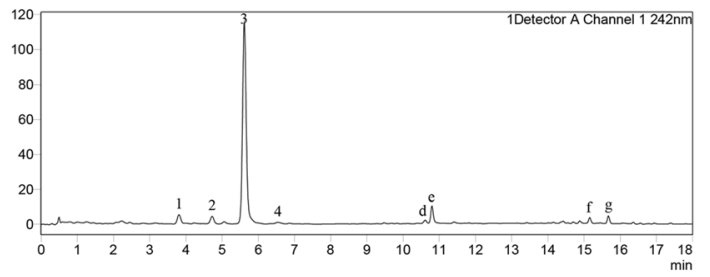

(i) $\mathrm{mv}$

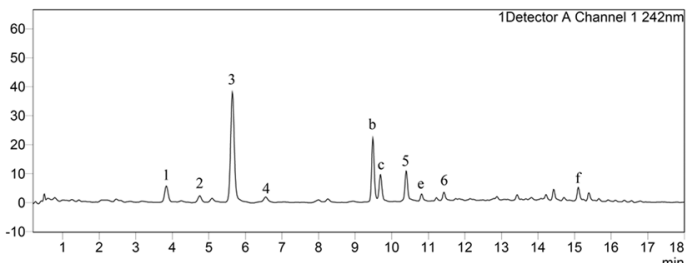

(b) $\mathrm{mv}$

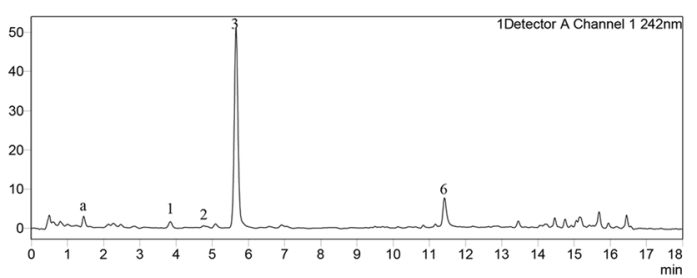

(d) $\mathrm{m}$

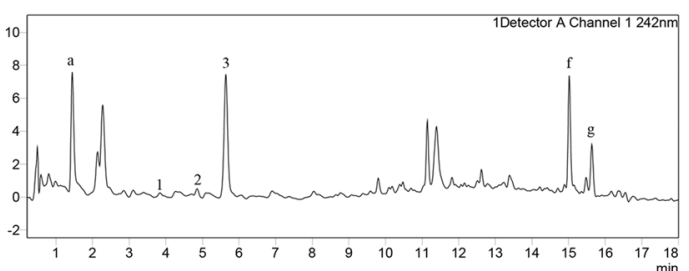

(f) $m v$

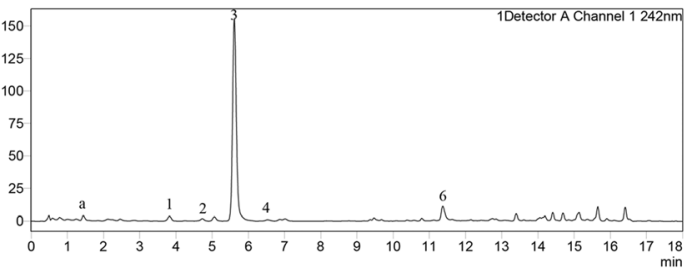

(h) $\mathrm{mv}$

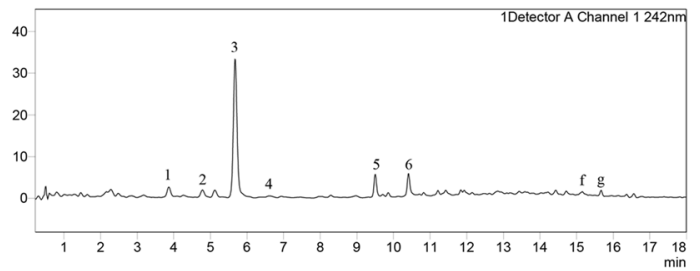

Fig. 4 UPLC profiling of G. rigescens. (1. loganic acid; 2. swertiamarin; 3. gentiopicroside; 4. sweroside; 5. isoorientin; 6. isovitexin; a: callus; b: BP-stem; c: BP-leaf; d: RP-root; e: RP-stem; f: RP-leaf; $\mathbf{g}$ : MP-root h: MP-stem; i: MP-leaf)

$\mathrm{b}$ and $\mathrm{c}$ could be tentatively assigned as flavonoids while peak $\mathrm{d}$-g were iridoid glycosides. In mass spectra of isoorientin and isovitexin (Additional file 1: Figure S1, see supplement data), the characteristic neutral loss of 90 and $120 \mathrm{Da}$ are correspond to $C$-glycosidic structure, which could be considered as diagnosed markers. Interestingly, the mass data of peak b and $\mathrm{c}$ (Additional file 1: Figure S1) were highly similar with isoorientin and isovitexin, respectively. Besides, the neutral loss of $162 \mathrm{Da}$, the feature of glucosyl group, 
were detected in both peak $\mathrm{b}$ and $\mathrm{c}$. Therefore, peak b $\left(\mathrm{m} / z \quad 593.1513 \quad[\mathrm{M}-\mathrm{H}]^{-}, \quad \mathrm{C}_{27} \mathrm{H}_{30} \mathrm{O}_{15}\right)$ and $\mathrm{c}(\mathrm{m} / \mathrm{z}$ $611.1503[\mathrm{M}-\mathrm{H}]^{-} \mathrm{C}_{27} \mathrm{H}_{30} \mathrm{O}_{16}$ ) were the $\mathrm{O}$-glycosidic derivatives of isoorientin and isovitexin, respectively.

Furthermore, a neutral loss of $136 \mathrm{Da}$ which may correspond to the loss of a dihydroxy benzoyl group via a classical McLafferty-type rearrangement (Tan et al. 1996; $\mathrm{Xu}$ et al. 2009b) was observed in mass spectrum of peak $\mathrm{d}$-g. By further analyzing the mass data, peak d-g were dihydroxy benzoyl iridoid glycosides. Their mass spectrum and fragmentation pattern are shown in Additional file 1: Figure S2. In mass spectrum of peak a, the product ions at $m / z 153$ and 109 were correspond to losing a glucose $(162 \mathrm{Da})$ and carboxyl group (44 Da), indicating peak a $\left(\mathrm{m} / \mathrm{z} 315.0832[\mathrm{M}-\mathrm{H}]^{-} \mathrm{C}_{13} \mathrm{H}_{16} \mathrm{O}_{9}\right)$ were tentatively assigned as $O$-glucosyl-dihydroxy benzoyl acid (Additional file 1: Figure S3) (Xu et al. 2009a). All compounds but peak 1-6 were identified by matching corresponding mass data in previous studies.

\section{Method validation and quantification}

Standard solutions of each compounds with seven different concentrations were prepared individually in methanol and were injected into LC-MS/MS system for generation of external standard calibration curves. Calibration curves for each compound were performed by plotting the peak area (y) against the concentrations $(\mathrm{x}, \mu \mathrm{g} / \mathrm{ml})$. The correlation coefficients $\left(\mathrm{R}^{2}\right)$ of each calibration curve were more than 0.9992. The limits of detection (LOD) and quantification (LOQ), S/N (signal-to-noise ratio) of 3 and 10, were determined by serial dilution of each standard solution using the described conditions. The LOD and LOQ for UV detector and mass analyzer, together with standard calibration curves of each standards, were listed in Table 1.
Precision was evaluated by intra- and inter-day variation which determined by analyzing mixed standard solutions with known concentration six times within a day and on three consecutive days in triplicate. The intra- and inter-day precision of peak area (expressed in terms of \%RSD) were in the range of $0.76-2.27 \%$. Accuracy was validated by recovery test performed by accurately adding three different amounts (low, medium and high spike) of each standard to the crude sample. The recovery rates of six standards were ranged from 97.3-102.6 \% and their RSD values were less than $3 \%$. Then, six independently samples analysed by repeating the described produce of sample preparation under this chromatographic condition were used to investigate the repeatability. These results are displayed in Table 2.

In this study, the identification of standards in the chromatogram was confirmed by retention times and ion pairs determined by MRM. Quantification depended on the external standard method. The contents of the six standards in G. rigescens during different rowing stage are listed in Table 3. The results showed that the content of the six standards differed greatly in different stage. The lowest contents of all standards were found in callus. Gentiopicroside was the highest yield compound in the whole growth stage. The highest gentiopicroside yield was found in leaf of regenerated plantlet $(122.93 \pm 7.01 \mathrm{mg} / \mathrm{g})$, followed by root of mature plant $(96.78 \pm 8.54 \mathrm{mg} / \mathrm{g})$. Interestingly, gentiopicroside yield was reduced and tended to accumulate in root after regenerated plantlet. Furthermore, root did not contain the two flavonoids. The highest content of the two flavonoids were observed in leaf. For isoorientin, the highest content was found in mature plants. On the contrary, the highest isovitexin yield was detected in proliferation stage.

Table 1 Linear regression and MRM parameters of standards

\begin{tabular}{|c|c|c|c|c|c|c|c|}
\hline Analytes & Regression equation & Linearity range $(\mu \mathrm{g} / \mathrm{mL})$ & $R^{2}$ & $\mathrm{LOD}(\mu \mathrm{g} / \mathrm{mL})$ & LOQ $(\mu \mathrm{g} / \mathrm{mL})$ & lons pairs & $\mathrm{CE}(\mathrm{eV})$ \\
\hline Loganic acid & $y=2099.0 x+19518.7$ & $50-500$ & 0.9996 & 0.34 & 1.01 & $421>375$ & 27 \\
\hline Loganic acid ${ }^{a}$ & $y=200150.0 x+2801.4$ & $0.05-5$ & 0.9997 & 0.004 & 0.022 & $421>213$ & 22 \\
\hline Swertiamarin & $y=2151.2 x+11429.0$ & $10-150$ & 0.9998 & 0.27 & 0.92 & $419>179$ & 18 \\
\hline Swertiamarin ${ }^{a}$ & $y=169057.0 x+3753.2$ & $0.1-5$ & 0.9995 & 0.005 & 0.027 & $419>141$ & 23 \\
\hline Gentiopicroside & $y=1494.4 x+21722.0$ & $5-1500$ & 0.9995 & 0.23 & 1.03 & $401>197$ & 14 \\
\hline- & - & - & - & - & - & $401>89$ & 21 \\
\hline Sweroside ${ }^{a}$ & $y=292679.0 x+5034.6$ & $0.5-10$ & 0.9992 & 0.008 & 0.037 & $359>197$ & -31 \\
\hline- & - & - & - & - & - & $359>127$ & -10 \\
\hline Isoorientin & $y=2945.4 x-6172.4$ & $5-250$ & 0.9993 & 0.57 & 2.33 & $447>327$ & 27 \\
\hline Isoorientin ${ }^{a}$ & $y=278957.0 x+4532.8$ & $0.05-5$ & 0.9994 & 0.007 & 0.041 & $447>357$ & 23 \\
\hline Isovitexin & $y=1979.7 x+19518.7$ & $5-250$ & 0.9998 & 0.63 & 1.97 & $431>311$ & 24 \\
\hline Isovitexin ${ }^{a}$ & $y=409813.0 x+1523.7$ & $0.05-5$ & 0.9996 & 0.007 & 0.037 & $431>331$ & 22 \\
\hline
\end{tabular}

-:not mentioned

${ }^{\mathrm{a}}$ :determination by MRM 
Table 2 Precision accuracy and repeatability (\%RSD) of this method

\begin{tabular}{|c|c|c|c|c|c|c|c|}
\hline \multirow[t]{3}{*}{ Analytes } & \multicolumn{3}{|c|}{ Intra-day RSD\% } & \multirow{3}{*}{$\begin{array}{l}\text { Inter-day RSD\% } \\
\mathrm{Pa}_{\mathrm{a}}\end{array}$} & \multirow{2}{*}{\multicolumn{2}{|c|}{ Accuracy $(n=9)$}} & \multirow{3}{*}{$\begin{array}{l}\text { Repeatability } \\
(n=6) \\
\text { RSD\% }\end{array}$} \\
\hline & \multirow{2}{*}{$\begin{array}{l}\text { Day } 1 \\
\mathrm{~Pa}\end{array}$} & \multirow{2}{*}{$\begin{array}{l}\text { Day } 2 \\
\mathrm{~Pa}\end{array}$} & \multirow{2}{*}{$\begin{array}{l}\text { Day } 3 \\
\mathrm{~Pa}\end{array}$} & & & & \\
\hline & & & & & Mean recovery & RSD\% & \\
\hline \multicolumn{8}{|l|}{ UV detector } \\
\hline Loganic acid & 1.13 & 2.09 & 2.36 & 1.58 & $102.6 \%$ & 1.93 & 1.79 \\
\hline Swertiamarin & 1.53 & 1.72 & 1.97 & 1.46 & $101.8 \%$ & 2.62 & 1.88 \\
\hline Gentiopicroside & 1.74 & 1.85 & 2.27 & 1.38 & $98.7 \%$ & 1.77 & 1.69 \\
\hline Isoorientin & 1.22 & 2.21 & 2.33 & 2.25 & $102.2 \%$ & 2.56 & 1.71 \\
\hline Isovitexin & 1.27 & 1.85 & 1.76 & 1.64 & $97.3 \%$ & 2.13 & 2.07 \\
\hline \multicolumn{8}{|l|}{ MRM } \\
\hline Loganic acid & 1.26 & 1.58 & 1.71 & 1.57 & $99.3 \%$ & 1.99 & 1.86 \\
\hline Swertiamarin & 1.45 & 1.31 & 1.67 & 1.78 & $100.9 \%$ & 1.86 & 1.22 \\
\hline Sweroside & 1.48 & 1.37 & 1.94 & 0.76 & 101.4 & 1.78 & 1.85 \\
\hline Isoorientin & 0.95 & 1.08 & 1.88 & 1.21 & $98.7 \%$ & 2.14 & 1.29 \\
\hline Isovitexin & 1.66 & 1.33 & 1.92 & 1.59 & $98.9 \%$ & 1.98 & 2.24 \\
\hline
\end{tabular}

$\mathrm{P}_{\mathrm{a}}$ : peak areas

\section{Discussions}

According to the results of comparative analysis, the distribution and accumulation of metabolites are associated with plant growth. The whole metabolome in different stage are significantly different. The reason might be attributed to (1) elicitors in medium and (2) environment conditions such as soil, sun exposure time and rainfall, which could also result in fluctuation on the distribution and accumulation of metabolites after transplant (Manukyan 2011; Marsh et al. 2014; Xie et al. 2011). Additionally, the proportion among metabolites vary with plant growth. The proportion of peak a (O-glucosyldihydroxy benzoyl acid) in chromatogram gradually reduce with the increase of other metabolites. Based on the identification of peak d-g, O-glucosyl-dihydroxy benzoyl acid may transformed into dihydroxy benzoyl iridoid glycosides via esterification of iridoid glycosides. The obvious differences are observed in chromatogram of leaf during different stage especially for peak b and c
(O-glycosidic derivatives of isoorientin and isovitexin) which only is detected in mature plant. These result implied that the synthesis and transform of metabolites with complex molecular structure tend to be in mature plant with more abundant substance when compared with plant during in proliferation stage.

Gentiopicroside is the characteristic compound with the highest amount in G. rigescens, which serve as standard for quality control of G. rigescens (State Pharmacopoeia Commission 2010; Wang et al. 2012). Combined with previous study on Gentiana scabra (Huang et al. 2014), another raw materials of Gentianae Radix et Rhizoma, 1.8 higher-fold gentiopicroside content was observed in sample of hair root culture than plants grown in greenhouse. Although leaf in regenerated plantlet contains the highest gentiopicroside yield in this study, it can only be used for industrial extraction of gentiopicroside rather than medical application because the efficacy of herb medicine, to large extend, is derived from synergistic effect of metabolites.

Table 3 Mean Contents $(\mathrm{mg} / \mathrm{g})$ of six standards $(n=6)$

\begin{tabular}{lllllll}
\hline Analytes & Loganic acid & Swertiamarin & Gentiopicroside & Sweroside & Isoorientin & Isovitexin \\
\hline Callus & $0.053 \pm 0.002^{\mathrm{a}}$ & $0.017 \pm 0.001^{\mathrm{a}}$ & $0.35 \pm 0.25$ & $0.05 \pm 0.001^{\mathrm{a}}$ & $0.013 \pm 0.002^{\mathrm{a}}$ & $0.075 \pm 0.01^{\mathrm{a}}$ \\
BP-stem & $3.12 \pm 0.17$ & $1.66 \pm 0.22$ & $45.11 \pm 2.21$ & $0.06 \pm 0.001^{\mathrm{a}}$ & $0.016 \pm 0.002^{\mathrm{a}}$ & $2.25 \pm 0.62$ \\
BP-leaf & $3.73 \pm 0.28$ & $2.78 \pm 0.44$ & $85.13 \pm 3.93$ & $0.81 \pm 0.04^{\mathrm{a}}$ & $0.021 \pm 0.002^{\mathrm{a}}$ & $2.17 \pm 0.58$ \\
RP-root & $0.034 \pm 0.005^{\mathrm{a}}$ & $0.038 \pm 0.005^{\mathrm{a}}$ & $0.55 \pm 0.03$ & $0.03 \pm 0.007^{\mathrm{a}}$ & - & - \\
RP-stem & $3.66 \pm 0.02$ & $2.65 \pm 0.02$ & $62.13 \pm 5.66$ & $0.36 \pm 0.03^{\mathrm{a}}$ & $0.036 \pm 0.001^{\mathrm{a}}$ & $2.87 \pm 0.72$ \\
RP-leaf & $4.55 \pm 0.72$ & $2.83 \pm 0.14$ & $122.93 \pm 7.01$ & $0.41 \pm 0.04^{\mathrm{a}}$ & $0.047 \pm 0.002^{\mathrm{a}}$ & $3.07 \pm 0.33$ \\
MP-root & $8.52 \pm 0.59$ & $6.16 \pm 0.72$ & $96.78 \pm 8.54$ & $0.27 \pm 0.07^{\mathrm{a}}$ & - & - \\
MP-stem & $4.90 \pm 0.11$ & $3.06 \pm 0.25$ & $17.58 \pm 3.21$ & $0.46 \pm 0.02^{\mathrm{a}}$ & $1.58 \pm 0.32$. & $0.83 \pm 0.02$ \\
MP-leaf & $5.51 \pm 0.21$ & $4.11 \pm 0.21$ & $26.05 \pm 4.33$ & $1.16 \pm 0.13^{\mathrm{a}}$ & $2.84 \pm 0.52$ & $0.85 \pm 0.22$ \\
\hline
\end{tabular}

a:Determination by MRM 
The variation on the accumulation of gentiopicroside in different stage is present in Fig. 5a. An interesting phenomenon that gentiopicroside content in root is significant raised with evidently decrease in leaf and stem, when the root start to regenerate. During this stage, root growth rate is far higher than in stem and leaf with the occurrence of gentiopicroside growth, which could be consistent with the results of specifictissue analysis where the distribution of secondary metabolites vary in different tissue. Moreover, gentiopicroside in aerial parts may translocate into root or transform into other metabolites when the root start to regenerate.

In leaf, a significant negative correlation is found between isoorientin and isovitexin (Fig. 5b). From the biosynthetic pathway of view, it can be explain that these two flavonoids are competitive relationship in a common biosynthetic pathway. Additionally, isovitexin can also be considered as a precursor of isoorientin and $O$-glycosidic isovitexin in this pathway. This may explain why the isovitexin first increase during proliferation stage, and then decrease.

\section{Conclusions}

In the present study, the combination use of FT-IR, LCUV-MS/MS and chemometrics was designed for investigation of the variation on metabolites during different growing stage of G. rigescens. For whole metabolome, the molecular structure-analyte relationships are significantly different between plants during proliferation stage and mature plants according to FT-IR analysis. Combined with LC-UV-MS/MS, mature plants contains more abundant secondary metabolites than plants during proliferation stage, whereas the higher content of some characteristic metabolites like gentiopicroside and peak a are observed in plants during proliferation. Moreover, the distribution and accumulation of metabolites, together with biosynthetic pathway, are associated with plant growth and significantly vary during different growing stage. In practical application, the root in mature plants with rich chemical components could be of better quality for medicinal application, whereas leaf in regenerated plantlet would be used for industrial extraction
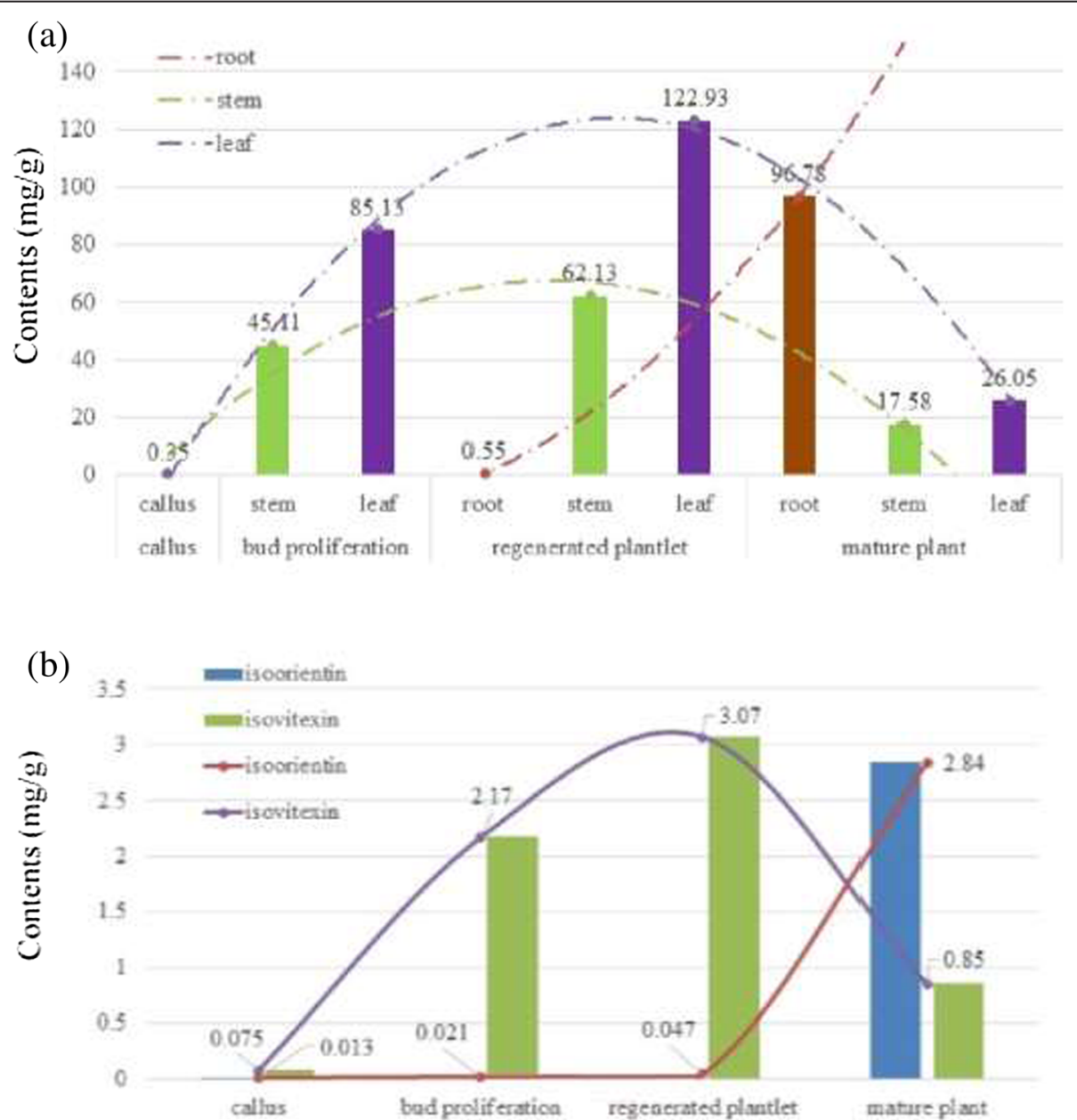

Fig. 5 a: Determination of gentiopicroside in different sample. b: Determination of isoorientin and isovitexin in leaf during different stage 
of gentiopicroside. These results provide evidence for reasonable exploitation and distinct usage of G. rigescens during different growing stage.

\section{Additional file}

Additional file 1: Figure S1. Mass spectrum of isoorientin, isovitexin, peak b and peak c. Figure S2. Mass spectrum of peak d-g. Figure S3. Mass spectrum of peak a. Table S1. Fuzz identification of characteristic peaks in UPLC profiling by mass spectrometry.

\section{Abbreviations}

BP: Bud proliferation; FT-IR: Fourier transform infrared spectroscopy; LOD: The limits of detection; LOQ: The limits of quantification; LC-MS/MS: Liquid chromatography tandem mass spectrometry; MP: Mature plant; MRM: Multiple reaction monitoring; RP: Regenerated plantlet.

\section{Competing interests}

The authors declare that they have no competing interests.

\section{Authors' contributions}

PY conducted all laboratory experiments, data analysis and partial interpretation of results under the guidance of $\mathrm{HH}-\mathrm{Y}$ and $\mathrm{WY}-\mathrm{Z}$, who designed the study. ZJ and $Z Y-L$ are responsible for the grammar checking of the paper and finalized the manuscript. All authors have read and approved the final manuscript.

\section{Acknowledgments}

This work was supported by the National Natural Science Foundation of China (81260608 and 31260077), Key Project of Yunnan Provincial Natural Science Foundation (2013FC006), and the Yunnan Provincial Natural Science Foundation (2013FZ150 and 2013FD066).

Received: 26 March 2015 Accepted: 18 May 2015

Published online: 27 May 2015

\section{References}

Chuang YK, Yang IC, Lo YM, Tsai CY, Chen S (2014) Integration of independent component analysis with near-infrared spectroscopy for analysis of bioactive components in the medicinal plant Gentiana scabra Bunge. J Food Drug Anal 22:336-344

Gao L, Li J, Qi J (2010a) Gentisides A and B, two new neuritogenic compounds from the traditional Chinese medicine Gentiana rigescens Franch. Bioorgan Med Chem 18:2131-2134

Gao L, Xiang L, Luo Y, Wang G, Li J, Qi J (2010b) Gentisides C-K: Nine new neuritogenic compounds from the traditional Chinese medicine Gentiana rigescens Franch. Bioorgan Med Chem 18:6995-7000

Hall RD, Brouwer ID, Fitzgerald MA (2008) Plant metabolomics and its potential application for human nutrition. Physiol Plantarum 132:162-175

Hilbert G, Temsamani H, Bordenave L, Pedrot E, Chaher N, Cluzet S, Delaunay JC, Ollat N, Delrot S, Mérillon JM, Gomès E, Richard T (2015) Flavonol profiles in berries of wild Vitis accessions using liquid chromatography coupled to mass spectrometry and nuclear magnetic resonance spectrometry. Food Chem 169:49-58

Hu Y, Kong W, Yang X, Xie L, Wen J, Yang M (2014) GC-MS combined with chemometric techniques for the quality control and original discrimination of Curcumae longae rhizome: Analysis of essential oils. J Sep Sci 37:404-411

Huang SH, Vishwakarma RK, Lee TT, Chan HS, Tsay HS (2014) Establishment of hairy root lines and analysis of iridoids and secoiridoids in the medicinal plant Gentiana scabra. Bot Stud 55:17-25

Jiang RW, Wong KL, Chan YM, Xu HX, But PPH, Shaw PC (2005) Isolation of iridoid and secoiridoid glycosides and comparative study on Radix Gentianae and related adulterants by HPLC analysis. Phytochemistry 66:2674-2680

Karoui R, Downey G, Blecker C (2010) Mid-infrared spectroscopy coupled with chemometrics: A tool for the analysis of intact food systems and the exploration of their molecular structure-quality relationships-a review. Chem Rev 110:6144-6168

Kumari T, Sharma C, Bajpai V, Kumar B, Srivastava M, Arya KR (2015) Qualitative determination of bioactive metabolites through Q-TOF LC/MS in different parts and undifferentiated cultures of UImus wallichiana Planchon. Plant Growth Regul 75:331-340

Kuzovkina IN, Prokof'eva MY, Umralina AR, Chernysheva TP (2014) Morphological and biochemical characteristics of genetically transformed roots of Scutellaria andrachnoides. Russ J Plant Physiol 61:697-706

Lohumi S, Lee S, Lee WH, Kim MS, Mo C, Bae H, Cho BK (2014) Detection of Starch Adulteration in Onion Powder by FT-NIR and FT-IR Spectroscopy. J Agri Food Chem 62:9246-9251

Manukyan A (2011) Effect of growing factors on productivity and quality of lemon catmint, lemon balm and sage under soilless greenhouse production: I. drought stress. Med Aromat Plant Sci Biotechnol 5:119-125

Marsh Z, Yang T, Nopo-Olazabal L, Wu S, Ingle T, Joshee N, Medina-Bolivar F (2014) Effect of light, methyl jasmonate and cyclodextrin on production of phenolic compounds in hairy root cultures of Scutellaria lateriflora. Phytochemistry 107:50-60

Pan Y, Shen T, Pan J, Xiao D, Li Z, Li W, Wang YZ (2014) Development and validation of a UPLC-MS/MS method for the simultaneous determination and detection of four neuritogenic compounds in different parts of Gentiana rigescens Franch using multiple reaction monitoring and precursor ion scanning. Anal Methods 6:1782-1787

Pan Y, Shen T, Zhang J, Zhao YL, Wang YZ, Li WY (2015a) Simultaneous determination of six index constituents and comparative analysis of four ethnomedicines from genus Gentiana using a UPLC-UV-MS method. Biomed Chromatogr 29:89-96

Pan Y, Zhang J, Shen T, Zuo ZT, Jin H, Wang YZ, Li WY (2015b) Optimization of ultrasonic extraction by response surface methodology combined with UFLC-UV method for determination of four iridoids in Gentiana rigescens. J Food Drug Anal doi: 10.1016/j.jfda.2014.11.002

State Pharmacopoeia Commission (2010) Chinese Pharmacopoeia, 2010th edn. Chemistry and Industry Press, Beijing, pp 89-90

Su P, Cheng Q, Wang X, Cheng X, Zhang M, Tong Y, Li F, Gao W, Huang LQ (2014) Characterization of eight terpenoids from tissue cultures of the Chinese herbal plant, Tripterygium wilfordii, by high-performance liquid chromatography coupled with electrospray ionization tandem mass spectrometry. Biomed Chromatogr 28:1183-1192

Tan RX, Wolfender JL, Zhang LX, Ma WG, Fuzzati N, Marston A, Hostei'itman K (1996) Acyl secoiridoids and antifungal constituents from Gentiana macrophylla. Phytochemistry 42:1305-1313

Wang YM, Xu M, Wang D, Zhu HT, Zhang YJ, Yang CR (2012) Review on "Long-Dan", one of the traditional Chinese medicinal herbs recorded in Chinese Pharmacopoeia. Nat Prod Bioprospect 2:1-10

Wink M (2003) Evolution of secondary metabolites from an ecological and molecular phylogenetic perspective. Phytochemistry 64:3-19

Won TH, Liao L, Kang SS, Shin J (2014) Simultaneous analysis of furfural metabolites from Rehmanniae radix preparata by HPLC-DAD-ESI-MS. Food Chem 142:107-113

Xie CX, Suo FM, Jia GL, Song JY, Huang LF, Chen S (2011) Correlation between ecological factors and ginsenosides. Acta Ecol Sin 31:7551-7563

Xu M, Wang D, Zhang YJ, Yang CR (2006) A new secoiridoidal glucoside from Gentiana rigescens (Gentianaceae). Acta Botanica Yunnanica 28:669-672

Xu M, Wang D, Zhang YJ, Yang CR (2007) Dammarane triterpenoids from the roots of Gentiana rigescens. J Nat Prod 70:880-883

Xu M, Yang CR, Zhang YJ (2009a) Minor antifungal aromatic glycosides from the roots of Gentiana rigescens (Gentianaceae). Chinese Chem Lett 20:1215-1217

Xu M, Zhang M, Zhang YJ, Yang CR (2009b) New acylated secoiridoid glucosides from Gentiana straminea (Gentianaceae). Helv Chim Acta 92:321-327

Yu C, Wang CZ, Zhou CJ, Wang B, Han L, Zhang CF, Wu XH, Yuan CS (2014) Adulteration and cultivation region identification of American ginseng using HPLC coupled with multivariate analysis. J Pharmaceut Biomed Anal 99:8-15

Zhao Y, Zhang J, Yuan T, Shen T, Li W, Yang S, Hou Y, Wang YZ, Jin H (2014) Discrimination of wild Paris based on near infrared spectroscopy and high performance liquid chromatography combined with multivariate analysis. PloS One 9, e89100 